\title{
A Novel Co-existence Algorithm for Unlicensed Variable Power Devices
}

\author{
Durga P. Satapathy \\ Sprint, d.p.satapathy@ieee.org \\ www.contrib.andrew.cmu.edu/ dsaq/home.html
}

\author{
Jon M. Peha \\ Professor, Carnegie Mellon University \\ peha@stanfordalumni.org; http://www.ece.cmu.edu/ peha/
}

\begin{abstract}
In unlicensed spectrum, any device is free to transmit without a license. Such spectrum has significant benefits, but serious challenges must first be overcome. Foremost is the risk of drastic performance degradation due to a lack of incentive to conserve shared resources. Previous work has identified this problem for devices that transmit for longer duration than necessary. This paper demonstrates this problem for devices that always transmit at maximum power to maximize throughput. For devices that can vary transmission power, the problem is solved if devices reduce transmission power when received interference exceeds defined thresholds. We propose a co-existence algorithm to optimize system throughput when each of two such devices can transmit up to the maximum power allowed on a given channel. We show device performance with current unlicensed band regulations is rarely optimal, and that the proposed algorithm is better.
\end{abstract}

\section{INTRODUCTION}

In unlicensed spectrum, any device is free to transmit without a license that implies exclusive access. Although most spectrum has traditionally been licensed [1], the Federal Communications Commission (FCC) has created several unlicensed bands, such as the Industry, Science and Medicine (ISM) bands, the Unlicensed Personal Communication Services (UPCS) band [2], the Unlicensed National Information Infrastructure (UNII) band [3], and the Millimeter Wave band [4]. The UPCS band is governed by a Spectrum Etiquette (known as the UPCS etiquette) [2,5], which is a set of rules regulating access to spectrum and its usage. Unlicensed spectrum has several benefits. It promotes spectrum sharing, and furthers experimentation and innovation. It facilitates mobility of wireless applications, as no licenses are needed for new locations. It is also suitable for smart environments [6], wherein intelligent devices interact with each others and users, sending user needs and acting accordingly. Three challenges must be overcome to realize such benefits. First, there may be mutual interference, as devices can transmit at will. Second, enforcing efficient utilization is difficult as applications using unlicensed bands may vary greatly. Third, there is little incentive to conserve shared spectrum. Thus, designers may adopt a greedy approach, where the more a device wastes shared spectrum to improve its performance, the more it is greedy. If this is common, the shared resource will be of little use. This phenomenon, referred to as a Tragedy of the Commons [7], made the Citizen Band radio service unusable in crowded regions, where users wasted spectrum with high-power transmitters. As the resources consumed by a device depend on transmission duration, bandwidth, and power, it may be greedy in any of these dimensions.

Previous work [8-10] has shown that greed in transmission duration can cause poor spectrum utilization. This paper demonstrates the same problem due to greed in the power dimension. Although all unlicensed bands enforce power limits to reduce interference, without any incentive to reduce power below the limit, greedy devices may transmit at maximum power. Given information (such as power, offered load, and distance) about other devices sharing spectrum, parameters that maximize system throughput can be determined. Without such explicit information, these parameters can only be chosen by an etiquette using available information, e.g. local noise and received power. Etiquette design is complicated by the diversity of devices. Some devices can vary transmission power, and some cannot. Also, power limits can vary from device to device. For devices that can vary transmission power, we propose the Sharing etiquette that avoids a Tragedy of the Commons. This etiquette optimizes system throughput when each of two such devices can transmit at the maximum power allowed on a channel, and optimizes individual throughput for isolated devices as well.

We use the following approach for performance comparison: We assume devices transmit at powers maximizing individual device throughput. We identify powers at which devices reach equilibrium, and compare system and individual throughputs at each equilibrium with the optimal throughput, throughput with UPCS etiquette, and with no etiquette. We show performance with current regulations in unlicensed bands is rarely optimal, and that the proposed etiquette performs better.

Section 2 presents our model to analyze greed in transmission power. Section 3 covers performance in unlicensed bands without an etiquette. Section 4 defines optimal performance of two devices sharing spectrum. Sections 5 and 6 discuss the UPCS and the Sharing etiquette respectively. Section 7 compares performance of existing and proposed etiquettes. Section 8 presents our conclusions. 


\section{THE MODEL SCENARIO}

Our model has two wireless networks, each with two cooperating elements: a device and its basestation. Elements belonging to different networks do not cooperate. The networks share a single channel of fixed bandwidth $\mathrm{B}$, as in one-way systems and in either the uplink or the downlink of two-way systems. Without loss of generality, we consider devices transmitting to their basestations. The path loss between Device $i$ and Basestation $i$ is given by the propagation factor $\beta_{i}$, and the path loss between Basestation $i$ and Device $j$ is given by the propagation factor $\alpha_{i}$. We assume symmetry in propagation loss from one network to another, i.e. $\alpha_{i}=\alpha_{j}=\alpha$. Since there will be devices that cannot determine noise and propagation factors, system designers must use estimated typical values. Thus, the etiquettes function as if each network has the same noise, and $\beta_{i}=\beta_{j}=\beta$, where $\beta$ is the propagation factor based on anticipated path loss. The propagation factors $\alpha$ (or $\beta$ ) decrease with distance as dictated by the path loss model. Device $i$ transmits at power $0 \leq P_{i} \leq \gamma_{i} P_{\max }$, where $P_{\max }$ denotes the maximum transmission power allowed on the channel, and $\gamma_{i} P_{\max }$ denotes the power limit of Device $i$. With Device $j$ at power $P_{j}$, Basestation $i$ receives power $R_{i}=N+\alpha P_{j}$. We assume that Device i either knows or can reasonably estimate the received power $R_{i}=N+\alpha P_{j}$. Thus, the signal-to-noise ratio (SNR) at Basestation $i$ is $\phi_{i}=\beta P_{i} /\left(N+\alpha P_{j}\right)$, and Basestation $j$ has $\phi_{j}=\beta P_{j} /\left(N+\alpha P_{i}\right)$. We define $\phi_{S}=\beta P_{\max } / N$ to be the SNR for Device $i$ when it transmits in isolation at power $P_{\max }$. We assume each Device $i$ has message error probability $E_{i}=\exp \left(-c \phi_{i}\right)$ where $c$ is a constant, as is appropriate for DPSK (Differential Phase Shift Keying) or non-coherent FSK (Frequency Shift Keying) modulation. Device $i$ has offered load $G_{i}$ (the sum of loads from arriving and retransmitted messages) and throughput $S_{i}=G_{i}\left(1-E_{i}\right)=G_{i}\left(1-\exp \left(-c \beta P_{i} /\left(N+\alpha P_{j}\right)\right)\right.$.

We determine device performance by observing individual and system throughput as devices vary these parameters: powers $P_{1}$ and $P_{2}$, power limits $\gamma_{1} P_{\max }$ and $\gamma_{2} P_{\max }$, loads $G_{1}$ and $G_{2}$, and propagation factor $\alpha$. We observe the impact of these input parameters on throughputs $S_{1}, S_{2}$ and system throughput $S_{1}+S_{2}$, which are the output parameters for our model.

\section{DEVICE BEHAVIOR WITH NO ETIQUETTE}

With transmission powers $P_{i}$ and $P_{j}$ fixed, devices maximize throughputs at $G_{i}=G_{j}=1$, i.e., by transmitting all the time [11]. Furthermore, if $G_{i}=G_{j}=1$, devices maximize throughput by transmitting at maximum power [11]. Thus, devices would always transmit at maximum powers, and that is the only equilibrium. The device with higher power gets greater throughput. However, such greed can result in a Tragedy of the Commons. In scenarios where the path loss between a device and its basestation is large relative to the path loss between its basestation and the interfering device, device throughput can degrade drastically.

\section{OPTIMAL SYSTEM THROUGHPUT}

Previous work [11] has shown that throughput is optimized when both devices always transmit, and that it is optimal for at least one device to transmit at maximum power. We have observed the optimal behavior to occur in two modes [11], one at small $\alpha$ and the other at large $\alpha$ (with boundary $\alpha_{B}$ ), and is defined as follows:

Optimal Behavior: Without loss of generality, let devices have power limits $\gamma_{1} \geq \gamma_{2}$. When $\alpha<\alpha_{B}$, both devices transmit at maximum power. When $\alpha \geq \alpha_{B}$, Device 1 transmits at $\gamma_{1} P_{\max }$ and the other transmits at a lesser power $0 \leq P<\gamma_{2} P_{\max }$ where $d \alpha_{B} / d N>0$ and $d \alpha_{B} / d \gamma_{2}<0$. For $\gamma_{1}=\gamma_{2}$ and $N=0$, the boundary is given by $\alpha_{B}=c \beta$.

For devices with unequal power limits, it is optimal for the device with higher power limit to transmit at a power greater than that of the other device [11]. Thus, there is an inherent tradeoff between maximizing throughput and fairness for devices with unequal power limits.

\section{THE UPCS ETIQUETTE}

The UPCS etiquette specifies a power limit $P_{\max }=100 \sqrt{B} m W$, where $B$ is the bandwidth in MHz. The etiquette enforces a "Listen Before Talk" (LBT) rule, requiring devices to transmit only if the received power is below a threshold throughout a specified monitoring period. It also allows devices to increase the LBT threshold by a $\mathrm{dB}$ for each $\mathrm{dB}$ reduction from the maximum power allowed.

There are two ranges of $\alpha$ that characterize device behavior [11]. The first is $\alpha$ where devices transmit at maximum power, as Theorem 1 shows. (For $\gamma_{1}=\gamma_{2}=1$ this range is denoted by $\alpha:\left\{0, \alpha_{U P C S}\right\}$.) For all other $\alpha$, Theorem 2 (Appendix A) shows that one device always transmits below 
its power limit, and in some cases both devices transmit below their power limits even though the resulting performance is not optimal.

Theorem 1: For $\gamma_{1} \geq \gamma_{2}$ and $\alpha<\left(K / \gamma_{1} P_{\max }-N\right) / \gamma_{2} P_{\max }$, each device receives power less than its LBT threshold, where $K \cong 1585 N P_{\max }$. For proof see [11].

\section{THE SHARING ETIQUETTE}

The Sharing etiquette, designed to maximize system throughput for devices that can vary transmission power up to $P_{\max }$, is defined as follows:

Sharing Etiquette: A device can transmit at maximum power if received power $R$ is below threshold $T=N+\alpha_{S} P_{\max }$. When received power exceeds this threshold, the etiquette imposes a maximum power $P_{S} \leq P_{\max }$, which is a function of $R$. $P_{S}$ satisfies Equation 1, derived by maximizing system throughput with respect to $P_{S}$ with other device at $P_{\max }$. In practice, $P_{S}$ would be pre-computed. $\alpha_{S}$ is derived from Equation 1 by solving for $\alpha$ with $P_{S}=P_{\max }$.

$\phi_{1} \exp \left(-c \phi_{1}\right)=\phi_{2} \exp \left(-c \phi_{2}\right) \alpha P_{S} /\left(N+\alpha P_{S}\right)$ with

$\phi_{1}=\beta P_{S} /\left(N+\alpha P_{\text {max }}\right) \quad$ and $\quad \phi_{2}=\beta P_{\text {max }} /\left(N+\alpha P_{S}\right) \quad$ and $\alpha=(R-N) / P_{\max }$.

We now determine equilibria with the Sharing etiquette. Without loss of generality, devices are numbered such that $\gamma_{1} \geq \gamma_{2}$. For any $\alpha$, there can be either one or two stable equilibria. For $\alpha<\alpha_{S} / \gamma_{1}$, each device transmits at maximum power, and this is the sole equilibrium. For $\alpha_{S} / \gamma_{1} \leq \alpha<\alpha_{S} / \gamma_{2}$, there is only one equilibrium: Device 1 transmits at maximum power. For $\alpha \geq \alpha_{S} / \gamma_{2}$, either Device 1 or Device 2 has maximum power at equilibrium. For $\alpha \geq \alpha_{S} / \gamma_{2}$, equilibrium is also possible with both devices below maximum power, but this is unlikely. (The instant either device stops transmission, the other transmits at maximum power.)

Device performance is characterized by up to three regions of $\alpha$ (see Figure 1): For $\alpha: \alpha \leq \alpha_{S} / \gamma_{1}$, both devices transmit at maximum power. For $\alpha: \alpha_{S} / \gamma_{1} \leq \alpha \leq \alpha_{S} / \gamma_{2}$, Device 1 transmits at maximum power and Device 2 below its maximum power $P_{S}$, which decreases as $\alpha$ increases. Although system throughput is optimized, the etiquette is not fair to the devices in this region. For $\alpha: \alpha_{S} / \gamma_{2}<\alpha \leq 1$, it is equally likely for either device to transmit at maximum power and the other below maximum power. Device 2 can have

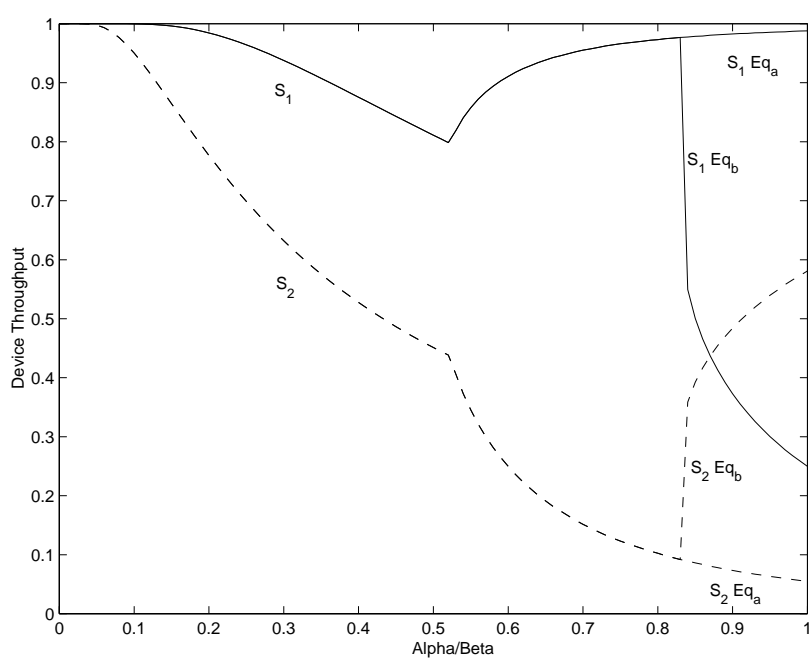

Fig. 1: System throughput at two equilibria with the Sharing etiquette and optimal throughput vs. propagation factor ratio $\alpha / \beta$ for $\gamma_{1}=1$,

$\gamma_{2}=0.6$ and $\phi_{S}=2.5 \times 10^{12}$. Equilibrium $E q_{a}$ corresponds to Device 1 at maximum power, and $E q_{b}$ to Device 2 at maximum power.

better throughput only when $\alpha>\alpha_{S} / \gamma_{2}$. However, with Device 2 at maximum power, Device 1 throughput falls sharply as $\alpha$ increases. Although the etiquette is fair, system performance can be far from optimal. This region does not exist if either device has $\gamma<\alpha_{S}$, as the other always receives power below its threshold. As devices with low power limits have poor performance relative to those with high power limits, multiple unlicensed bands (each with a smaller power limit range) might be better than a single band for devices with a wide range of power limits.

\section{PERFORMANCE COMPARISONS}

We compare performance of devices with the Sharing etiquette, the UPCS etiquette, and with no etiquette by evaluating the throughputs of devices at equilibrium. As Appendix B shows, the relative performance of etiquettes depends on $\sqrt{B} / \beta$. We therefore select various $\sqrt{B} / \beta$ values in a $100 \mathrm{MHz}$ band for etiquette comparison.

For $\gamma_{1}=\gamma_{2}=\gamma \leq \alpha_{S}$, devices always transmit at maximum powers (as received power at any $\alpha$ never exceeds threshold for either device). Thus, performance with the Sharing etiquette equals that with no etiquette. For $\gamma_{1}=\gamma_{2}=\gamma>\alpha_{S}$, the Sharing etiquette achieves the best performance. Performance with all but UPCS is always equal or better than with no etiquette for all $\alpha$. The UPCS etiquette is always suboptimal, even with $\beta$ for which the UPCS threshold is optimal. Figure 2 shows these results for $\gamma_{1}=\gamma_{2}=1$. 


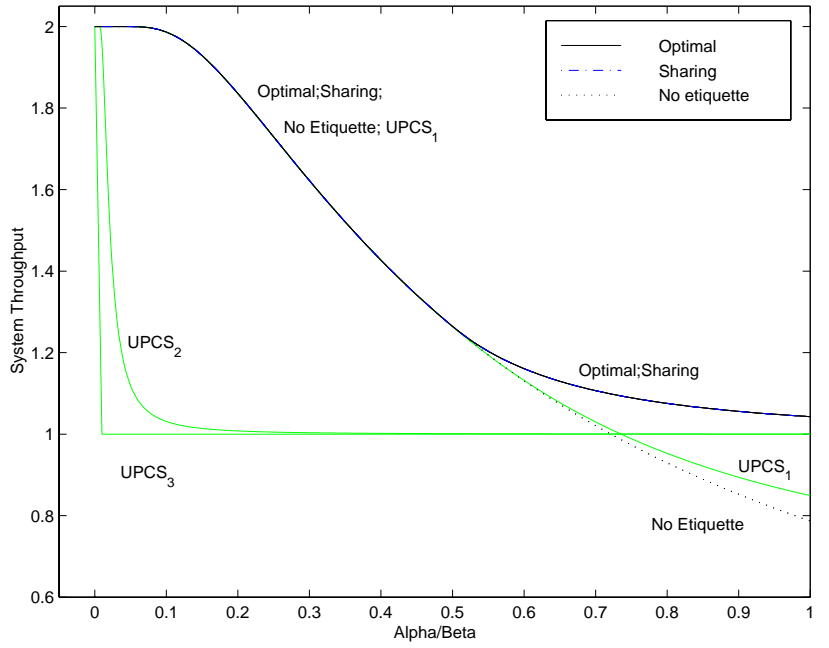

Fig. 2: System throughput vs. propagation factor ratio $\alpha / \beta$ with the Sharing etiquette, the UPCS etiquette, with no etiquette and the optimal case for $\gamma_{1}=\gamma_{2}=1$ for a $100 \mathrm{MHz}$ UNII band with $N / P_{\max }=4 \times 10^{-13}$. $\mathrm{UPCS}_{1}$ has $\beta=1.26 \times 10^{-9}$, $\mathrm{UPCS}_{2}$ has $\beta=10^{-6}$ and $\mathrm{UPCS}_{3}$ has $\beta=1$.

For devices with unequal power limits, the equilibria with Device 1 at maximum power and the equilibria with Device 2 at maximum power are equally likely with the Sharing and the UPCS etiquette. Figure 3 compares performance by averaging the throughputs at the two equilibria for $\gamma_{1}=1$ and $\gamma_{2}=0.6$. The Sharing etiquette is better than others over a wide range of $\alpha$, except for $\alpha: \alpha_{S} / \gamma_{2}<\alpha \leq 1$ where the device with higher power limit can transmit below maximum power. We conclude that the Sharing etiquette provides the best overall performance.

\section{CONCLUSION}

Unlicensed spectrum has several advantages. However, with little inherent incentive to conserve spectrum, designers may adopt greedy strategies, where the more a device wastes shared spectrum to improve performance, the more it is greedy. Devices may display greed in transmission duration, bandwidth, or transmission power. Previous work [8-10] has shown severe performance degradation due to greed in transmission duration, and suggested solutions. This paper explores greed in transmission power. We show that in bands with power limits only, devices would maximize throughput by always transmitting at maximum power, which also optimizes system throughput when devices are far apart. When devices are near, transmitting at maximum power leads to suboptimal performance. The solution lies in a properly designed etiquette (i.e. a set of rules regulating spectrum usage). This paper proposes the Sharing etiquette, which decreases the maximum power allowed as received power

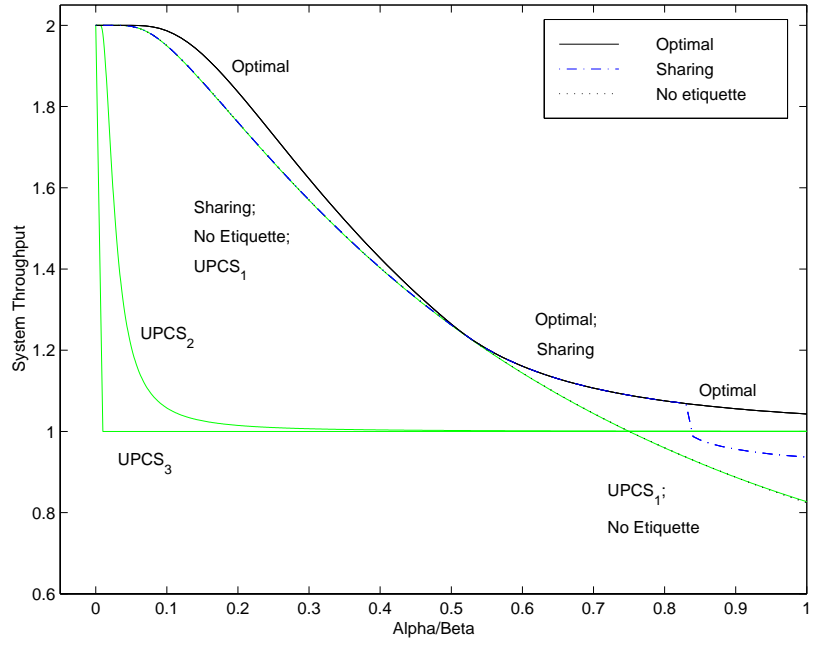

Fig. 3: System throughputs vs. propagation factor ratio $\alpha / \beta$ with the Sharing etiquette, the UPCS etiquette, no etiquette and the optimal case with $\gamma_{1}=1 ; \gamma_{2}=0.6$ for a $100 \mathrm{MHz}$ UNII band with $N / P_{\max }=4 \times 10^{-13}$.

$\mathrm{UPCS}_{1}$ has $\beta=1.26 \times 10^{-9}, \mathrm{UPCS}_{2}$ has $\beta=10^{-6}$ and $\mathrm{UPCS}_{3}$ has $\beta=1$.

increases beyond a threshold. This etiquette optimizes throughput of isolated devices, and system throughput as well when each of two devices can transmit at the maximum power allowed on a given channel. We demonstrate that this etiquette performs better than current regulations. We show system performance can be improved by discriminating between devices based on transmission power, and by creating multiple unlicensed bands, each band catering to devices with a small range of power limits.

\section{APPENDIX A}

Theorem 2: For $\alpha>\left(K / \gamma_{1} P_{\max }-N\right) / \gamma_{2} P_{\max } \quad$ where $K \cong 1585 N P_{\max }$, the following stable equilibria exist given $\gamma_{1} \geq \gamma_{2}$ and $P_{n}=-0.5 N / \alpha+0.5 \sqrt{N^{2}+4 \alpha K} / \alpha$ :

For $P_{n} \leq \gamma_{2} P_{\text {max }}$, there is one equilibrium $P_{1}=P_{2}=P_{n}$.

For $\gamma_{2} P_{\max }<P_{n}<\gamma_{1} P_{\max }$, there is one equilibrium $P_{2}=\gamma_{2} P_{\max }, P_{1}=K /\left(N+\alpha P_{2}\right)$.

For $P_{n} \geq \gamma_{1} P_{\max }$, there exist two stable equilibria $P_{1}=\gamma_{1} P_{\max }, P_{2}=\min \left\{\gamma_{2} P_{\max }, K /\left(N+\alpha P_{1}\right)\right\} \quad$ and $P_{1}=\min \left\{\gamma_{1} P_{\max }, K /\left(N+\alpha P_{2}\right)\right\}, P_{2}=\gamma_{2} P_{\max }$.

Proof: Let devices reach equilibrium at $P_{1}$ and $P_{2}$ for a given $\alpha$. As devices transmit at maximum power allowed, $P_{1}=K /\left(N+\alpha P_{2}\right)$ and $P_{2}=K /\left(N+\alpha P_{1}\right)$. Thus, $P_{1}=P_{2}=P$ where $\alpha P^{2}+N P-K=0$, i.e. $P_{m}=-0.5 N / \alpha+0.5 \sqrt{N^{2}+4 \alpha K} / \alpha<0$ and $P_{n}=-0.5 N / \alpha+0.5 \sqrt{N^{2}+4 \alpha K} / \alpha>0$. Thus the only 
equilibrium is $P_{1}=P_{2}=P_{n}=-0.5 N / \alpha+0.5 \sqrt{N^{2}+4 \alpha K} / \alpha$, which exists only if $P_{n} \leq \gamma_{2} P_{\max }$ (given $\gamma_{1} \geq \gamma_{2}$ ).

We now show $P_{1}=P_{2}=P n=K /\left(N+\alpha P_{n}\right)$ is a stable equilibrium. If Device 1 reduces power to $P_{1}<P_{n}, R_{1}=N+\alpha P_{n}=K / P_{n}<K / P_{1}$. Thus, Device 1 selects maximum allowed power $K /\left(N+\alpha P_{n}\right)=P_{n}$. If $P_{1}>P_{n}, R_{1}=N+\alpha P_{n}=K / P_{n}>K / P_{1}$. Device 1 stops transmitting and reduces power to $P_{1}=K /\left(N+\alpha P_{n}\right)=P_{n}$. If $\gamma_{2} P_{\max }<P_{n}<\gamma_{1} P_{\max }$, then there exists one stable equilibrium $P_{2}=\gamma_{2} P_{\max }, P_{1}=K /\left(N+\alpha P_{2}\right)$. This occurs when either device is already transmitting at maximum power before the other gains access. If $P_{1}=\gamma_{1} P_{\max }$, Device 2 has maximum allowed power $P_{2}=K /\left(N+\alpha P_{1}\right)$. Device 1 then receives power $R_{1}=N+\alpha K /\left(N+\alpha P_{1}\right)$. Device 1 can continue transmitting only if its received power is less than its threshold $T_{1}=K / P_{1}$, i.e., if $\alpha P_{1}^{2}+N P_{1}-K<0$, which is not true as $P_{1}=\gamma_{1} P_{\max }>P_{n}$. Device 1 will therefore reduce power as long as $P_{1}>P_{n}$, and Device 2 will increase power. As $\gamma_{2} P_{\max }<P_{n}$, devices reach equilibrium with $P_{2}=\gamma_{2} P_{\max }$ and Device 1 at $P_{1}=K /\left(N+\alpha P_{2}\right)$.

If $P_{n} \geq \gamma_{1} P_{\max }$, then devices can reach these two equilibria: $P_{1}=\gamma_{1} P_{\max }, P_{2}=\min \left\{\gamma_{2} P_{\max }, K /\left(N+\alpha P_{1}\right)\right\}$ and $P_{1}=\min \left\{\gamma_{1} P_{\max }, K /\left(N+\alpha P_{2}\right)\right\}, P_{2}=\gamma_{2} P_{\max } . \quad$ If $P_{1}=\gamma_{1} P_{\max }$, Device 2 selects $P_{2}=K /\left(N+\alpha P_{1}\right)$ and $R_{1}=N+\alpha K /\left(N+\alpha P_{1}\right)$. Device 1 can continue transmitting only if $\alpha P_{1}^{2}+N P_{1}-K<0$, which is true as $P_{1}=\gamma_{1} P_{\max }<P_{n}$. Thus, $P_{1}=\gamma_{1} P_{\max }, P_{2}=\min \left\{\gamma_{2} P_{\max }, K /\left(N+\alpha P_{1}\right)\right\}$ is one equilibrium. $P_{1}=\min \left\{\gamma_{1} P_{\max }, K /\left(N+\alpha P_{2}\right)\right\}, P_{2}=\gamma_{2} P_{\max } \quad$ from symmetry. These equilibria are stable, as one device is at maximum power and other at maximum power allowed.

\section{APPENDIX B}

For $P_{i}=\gamma_{i} P_{\max }$, the UPCS threshold is $T_{i} \cong 1585 N / \gamma_{i}$ with $N=k T B$, where $k$ is the Boltzman constant, $T$ is temperature in Kelvin, and $B$ is bandwidth in $\mathrm{Hz}$. For $\gamma_{i}=1, N+\alpha_{U P C S} P_{\max }=T_{i}$ i.e. $\alpha_{U P C S}=1584 N / P_{\max }$. With the UPCS etiquette, the maximum power allowed is $P_{\max }=10^{-4} \sqrt{B} . \quad$ Thus, $\quad \alpha_{U P C S}=1584 k T \sqrt{B} \times 10^{4}$, where $k T=-174 \mathrm{dBm} / \mathrm{Hz}$ at $T=290 \mathrm{Kelvin} . \quad$ Thus, $\quad \alpha_{U P C S}$ is independent of $\beta$. In contrast, $\alpha_{S}$ depends on $\beta$ (Equation
1). As $\alpha_{S} \cong 0.501 \beta, \quad \alpha_{U P C S}$ equals $\alpha_{S}$ when $\sqrt{B} / \beta=7.94 \times 10^{9}$, i.e. for $\beta \cong 1.26 \times 10^{-9}$ when $\mathrm{B}=100 \mathrm{MHz}$.

At $5 \mathrm{GHz}$ (with $46.4 \mathrm{~dB}$ loss at reference distance 1 meter) and a path loss modeled by a 3.0 propagation exponent, $\beta \cong 8.74 \times 10^{-10}$ corresponds to 29.6 meters, and $\beta \cong 1.26 \times 10^{-9}$ to 26.4 meters. For shorter distances, we consider $\sqrt{B} / \beta=10^{7}$ which equates to 2.84 meters.

\section{REFERENCES ${ }^{*}$}

[1] J. M. Peha, "Spectrum Management Policy Options," IEEE Communication Surveys, Fourth Quarter 1998, www.comsoc.org/pubs/surveys/4q98issue/pdf/Peha.pdf

[2] FCC, "Amendment of the Commission's Rules to Establish New Personal Communications Services," Memorandum Opinion and Order, FCC 94-144, Gen. Docket No. 90-314, June 9, 1994.

[3] FCC, "Amendment of the Commission's Rules to Provide for Operation of Unlicensed NII Devices in the $5 \mathrm{GHz}$ Frequency Range," Report \& Order, FCC 97-005, Gen. Docket No. 96-102, Jan. 9, 1997.

[4] M. J. Marcus, "Recent Progress in U.S. Millimeter Wave Spectrum Management Policy," IEEE MTT-S International Microwave Symposium Digest, vol. 2, pp. 505-7, June 1996.

[5] D. G. Steer, "Wireless Operation in the Unlicensed Band," Proc. Intl. Conf. on Universal Personal Communications, Sept. 1994, pp. 586-90.

[6] J. M. Peha, "Wireless Communications and Coexistence for Smart Environments," IEEE Personal Communications, Volume 7, Number 5, October 2000, pp. 66-68.

[7] G. Hardin, "The Tragedy of the Commons," Science, vol. 162,1968 , pp. 1243-1248.

[8] D. P. Satapathy and J. M. Peha, "Performance of Unlicensed Devices With a Spectrum Etiquette," Proceedings of IEEE Globecom, Nov. 1997, pp. 414-418.

[9] D. P. Satapathy and J. M. Peha, "Etiquette Modifications For Unlicensed Spectrum: Approach and Impact," Proceedings of the 48th Annual International IEEE Vehicular Technology Conference, May 1998, vol.1, pp. 272276.

[10] D. P. Satapathy and J. M. Peha, "Dangers of Excessive Resource Consumption in Unlicensed Spectrum," accepted for publication in ACM Wireless Networks.

[11] D. P. Satapathy and J. M. Peha, "A Novel Co-existence Strategy for Unlicensed Fixed-Power Devices," Proceedings of IEEE Wireless Communications and Networking Conference (WCNC), September 2000, vol.3, pp. 1403-1409.

* Most references are available at www.ece.cmu.edu/ peha/papers.html 